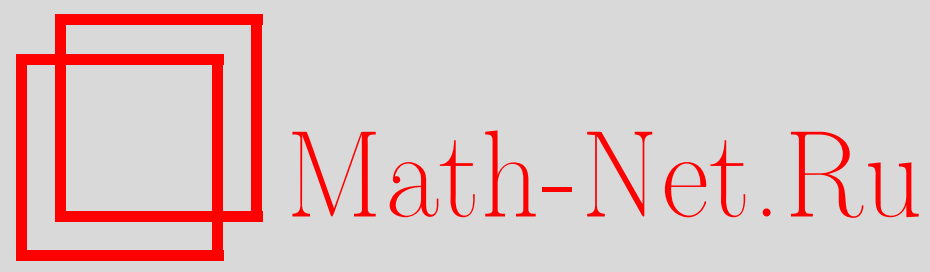

В. Г. Ильичев, Универсальные константы запаса и критерии отбора в переменной среде, Матем. заметки, 2001, том 70, выпуск 5, 691-704

DOI: https://doi.org/10.4213/mzm781

Использование Общероссийского математического портала Math-Net.Ru подразумевает, что вы прочитали и согласны с пользовательским соглашением http://www . mathnet.ru/rus/agreement

Параметры загрузки:

IP: 18.209 .158 .208

26 апреля 2023 г., $15: 42: 47$

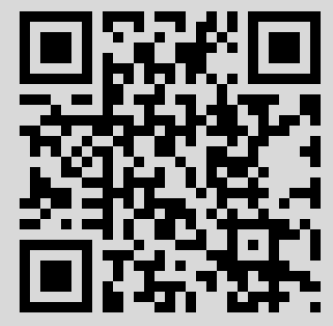




\section{УНИВЕРСАЛЬНЫЕ КОНСТАНТЫ ЗАПАСА И КРИТЕРИИ ОТБОРА В ПЕРЕМЕННОЙ СРЕДЕ}

\section{В.Г. Ильичев}

Разработан принцип наследования локальных свойств глобальньм отображением Пуанкаре для неавтономных динамических систем. Данный метод использован для нахождения критерия отбора в системе "близких" конкурентов: для конкурентного преимущества достаточно превосходить (по продуктивности) остальные популяции с запасом. При этом константа запаса остается равномерно ограниченной при увеличении количества конкурентов в сообществе.

Библиография: 10 названий.

Введение. В математической экологии актуальна проблема нахождения критериев отбора в сообществе конкурирующих популящий. Отметим, что в стабильной среде данная задача допускает простое решение, основанное на сравнении индивидуальных характеристик популяций. А именно, выживает популяция с наибольшей равновесной численностью [1]. В этой связи для периодической среды (например, при изменении температуры) естественно определить следующую индивидуальную характеристику: $X_{i}^{*}$ - средняя за период численность $i$-й популящии в ее равновесном режиме при отсутствии конкурентов. Пусть сообщество представлено $n$ конкурентами и для каждого из них известна соответствующая величина $X_{i}^{*}$. При каких ограничениях на величины $X_{1}^{*}$ и $X_{2}^{*}, \ldots, X_{n}^{*}$ можно гарантировать, что первая популяция вытесняет остальные? Оказывается, условие $X_{1}^{*}>X_{i}^{*}$ для всех $i>1$ вовсе не достаточно для доминирования первой популяции в таком сообществе. Так, при $n=2$ найдены "парадоксальные" модельные примеры, в которых $X_{1}^{*}>X_{2}^{*}$, но вторая популящия вытесняет первую [2]. Более правдоподобной представляется следующая

ГИПотеЗА. Для каждого $n$ существует константа запаса $Z(n)$ такая, что если $X_{1}^{*}>X_{i}^{*} \cdot Z(n)$ для всех $i=2, \ldots, n$, то в сообществе первая популяция вытесняет остальные.

Отметим, что функция $Z(n)$ может зависеть от выбора модельной схемы конкуренции. Пусть выбрана конкретная модельная схема - $n$-мерная система нелинейных дифференциальных уравнений с периодическими коэффициентами. Тогда здесь принципиальное значение имеет вопрос: является ли последовательность $\{Z(n)\}$ ограниченной или нет. Если она равномерно ограничена, то будем говорить, что существует универсальная (не зависящая от $n$ ) константа запаса $Z^{*}$.

Работа выполнена при поддержке Российского фонда фундаментальных исследований, грант № 00-01-00725. 
Ранее для модельной схемы Контуа была установлена “своя" универсальная константа запаса [3]. В этой связи целью данной статьи является доказательство существования универсальных констант запаса для широкого класса моделей конкуренции.

Последнее. Анализ нелинейных неавтономных систем дифференпиальных уравнений представляет собой непростую математическую задачу из-за трудностей с нахождением свойств глобального отображения Пуанкаре (сдвиг-отображение за период). С помощью теории так называемьх знак-инвариантных матриц (п. 2) обнаружена возможность переноса некоторых локальных (легко проверяемых) свойств моделей на отображение Пуанкаре. Так, в двумерных моделях конкуренции этот подход сразу приводит к цели (п. 3), а в многомерных (п. 4) - после “трюка" с обрашением времени.

1. Модели нейтральной конкуренции. Достаточно общая модель динамики численности популяции $(x)$ имеет вид [4]

$$
\dot{x}=x f(x, t)
$$

где $f(x, t)$ - гладкая функция и $T$-периодическая по переменной $t$. Здесь подразумевается, что второй аргумент функции $f$ учитывает действие периодических факторов среды.

Начальное значение $x^{0} \geqslant 0$ и ввиду специальной структуры (1.1) переменная $x$ будет неотрицательной и при всех $t>0$.

Самое главное, что функция $f$ отражает негативное действие внутренней конкуренции на скорость роста популяции, поэтому $\partial f / \partial x<0$ при всех $x>0$ и $t>0$. Будем предполагать, что при достаточно больших $x$ вьполняется условие $f(x, t)<0$. Данное неравенство "не позволяет" численности популяции неограниченно возрастать. С другой стороны, потребуем $f(0, t)>0$ хотя бы для некоторых $t$, иначе популящия однозначно вымирает.

Для описания динамики взаимодействия $n$ конкурентов традиционно используется система

$$
\dot{x}_{i}=x_{i} g_{i}\left(x_{1}, \ldots, x_{n}, t\right), \quad \text { где } i=1, \ldots, n,
$$

в которой каждая функция $g_{i}$ убывает по всем переменным $x_{1}, \ldots, x_{n}$.

Теперь рассмотрим сообшество “близких" (нейтральных) конкурентов. Известно, что отбор происходит именно в семействе близких популящий. Здесь понятие "близость" означает, что в системе (1.2) на каждую $i$-ю популяцию все популяции оказывают одинаковое конкурентное давление. Точнее, для всех точек $x$ из $\mathbb{R}_{+}^{n}$ и $t \geqslant 0$ вьполняются равенства

$$
\frac{\partial g_{i}}{\partial x_{1}}=\cdots=\frac{\partial g_{i}}{\partial x_{n}}
$$

для каждого $i$. Отсюда вьводимо соотношение [5]

$$
g_{i}\left(x_{1}, \ldots, x_{n}, t\right)=f_{i}\left(x_{1}+\cdots+x_{n}, t\right) .
$$

Предполагается, что каждая гладкая функция $f_{i}$ удовлетворяет прежним ограничениям (см. (1.1)) и, в частности, $\partial f_{i}(z, t) / \partial z<0$.

Для получения более или менее содержательных результатов следует указать вид вхождения периодических коэффициентов в правую часть системы (1.2). В этой связи ниже будет рассмотрен следующий довольно распространенньй класс моделей [6]

$$
\dot{x}_{i}=x_{i}\left[-1+\frac{\beta_{i}(t)}{V\left(x_{1}+\cdots+x_{n}\right)}\right],
$$


где $i=1, \ldots, n$. Здесь $\beta_{i}(t)$ - максимальная скорость роста в зависимости от $T$-периодических факторов среды; $\beta_{i}(t)$ - положительная $T$-периодическая функция. Заметим, что при очень низких скоростях роста в (1.3) происходит экспоненциальное уменьшение численности (т.е. скорость смертности равна -1).

Гладкая, положительная и возрастающая функция $V(z)$ учитывает негативное действие конкуренщии. Положим $V(0)=1$ и $V(\infty)=\infty$. Обозначим через $v$ обратную функцию к $V$. Очевидно, что $v(z)$ - монотонно возрастающая функция, определенная при $z \geqslant 1 ; v(1)=0$. Для $z<1$ доопределим $v(z)=0$.

Легко видеть, что переменные (1.3) как растут, так и убывают не быстрее соответствующих экспонент. Поэтому здесь решения продолжаются вперед и назад неограниченно. Обозначим через

$$
\lambda_{i}=\frac{1}{T} \int_{0}^{T} \beta_{i}(t) d t
$$

среднюю скорость коэффищиента роста $i$-го конкурента.

Величина $\lambda_{i}$ является характеристикой продуктивности $i$-го конкурента. Теперь проблему нахождения критерия отбора можно переформулировать следующим образом: на сколько $\lambda_{1}$ должно быть больше других $\left\{\lambda_{i}\right\}$, чтобы первая популящия вытеснила остальные?

2. Знак-инвариантные матрицы $\Sigma$ и монотонные отображения в $\mathbb{R}^{n}$. При исследовании глобальных свойств неавтономных моделей

$$
\dot{X}=F(X, t)
$$

ключевую роль играет сдвиг-отображение за период $(T)$ по траекториям системы $(2.1)$, т.е. $X^{T}=P\left(X^{0}\right)$. Когда задана конкретная система, то обычно не составляет труда нахождение тех или иных свойств (с точностью до малых $o(h))$ локального отображения

$$
X^{t+h}=L\left(X^{t}\right)=X^{t}+h F\left(X^{t}, t\right)
$$

где $h$ мало и $h>0$. Здесь актуальна задача: какие свойства локального отображения $(L)$ наследуются глобальньм отображением $(P)$ ?

Рассмотрим один из аспектов этой проблемы - сохранение свойств монотонности. Поясним, какие бывают “монотонности” у гладких отображений $P: \mathbb{R}^{n} \rightarrow \mathbb{R}^{n}$.

1) При одномерном отображении $P: \mathbb{R}^{1} \rightarrow \mathbb{R}^{1}$ характеристикой монотонности в точке $x$ является знак производной $d P(x) / d x$. В типичном случае здесь существуют лишш два варианта - плюс (т.е. возрастание) и минус (т.е. убывание).

2) При отображении $P: \mathbb{R}^{2} \rightarrow \mathbb{R}^{2}$ возникают 16 типичных вариантов, соответствующих знаковой структуре дифференциала $P$. Более формально, пусть $P$ имеет следующее покоординатное представление:

$$
\left(x_{1}, x_{2}\right) \rightarrow\left(P_{1}\left(x_{1}, x_{2}\right), P_{2}\left(x_{1}, x_{2}\right)\right)
$$

Тогда дифференциал $P$ в точке $\left(x_{1}, x_{2}\right)$ задается матрицей

$$
D P=\left(\begin{array}{ll}
\partial P_{1} / \partial x_{1} & \partial P_{1} / \partial x_{2} \\
\partial P_{2} / \partial x_{1} & \partial P_{2} / \partial x_{2}
\end{array}\right)
$$


3) Аналогично, в общем случае для $P: \mathbb{R}^{n} \rightarrow \mathbb{R}^{n}$ возникают $2^{m}$ типичных вариантов монотонности, где $m=n \times n$.

Поставим теперь следующий вопрос: пусть все $D L$ представляются одной и той же знаковой таблищей $\Sigma$ для всех $x$ и $t$. Когда $D P$ также описьвается той же таблицей $\Sigma ?$

Предлагаемое ниже решение опирается на понятие знак-инвариантных структур [7]. Это специальный вид знаковых $\left(c_{1}, \ldots, c_{n}\right)$-параметрических таблиц $\Sigma=\left(\sigma_{i j}\right)$ размера $n \times n$, элементы которых равны соответственно $\sigma_{i j}=c_{i} c_{j}$. Здесь каждьй параметр может принимать одно из двух значений $c_{i}= \pm 1$. Например, при $n=2$ существуют только две разные знак-инвариантные структуры:

$$
\Sigma(1,1)=\Sigma(-1,-1)=\left(\begin{array}{ll}
+1 & +1 \\
+1 & +1
\end{array}\right), \quad \Sigma(-1,1)=\Sigma(1,-1)=\left(\begin{array}{ll}
+1 & -1 \\
-1 & +1
\end{array}\right)
$$

Очевидно, матрицы с фиксированной знак-инвариантной структурой $\Sigma=\Sigma\left(c_{1}, \ldots, c_{n}\right)$ образуют полугрупу по умножению. Отсюда получаем "своеобразньй" принцип переноса.

УТВЕРЖДЕНИЕ 1. Если в (2.1) все DL принадлежсат знак-инвариантной струкmуре $\Sigma$, то и DP принадлежит той же $\Sigma$.

ДокАЗАТЕЛЬСтво. Воспользуемся методом “деления пополам" для сдвиг-отображений. Так, представим отображение $P$ как композицию двух “пополам"-отображений $\pi_{2} \circ \pi_{1}$ :

1) $\pi_{1}$ является сдвиг-отображением за время $[0, T / 2]$;

2) $\pi_{2}$ является сдвиг-отображением в интервале $[T / 2, T]$.

Очевидно, матрица $D P$ является произведением матриц $D \pi_{2}$ и $D \pi_{1}$.

Теперь предположим противное: знаковая структура $D P$ является “плохой", т.е. не относится к типу $\Sigma$. Поэтому хотя бы у одной из матриц $D \pi_{1}$ или $D \pi_{2}$ знаковая структура оказьвается “плохой". Пусть “плохой” матрицей будет, например, $D \pi_{1}$. Тогда разложим $\pi_{1}$ в композицию двух “пополам"-отображений и т.д.

Ясно, что такой процесс выбора "плохой” матрицы можно продолжать сколь угодно долго. Значит, существует достаточно малый временной интервал $[t, t+h]$, на котором сдвиг-отображение $\pi^{*}$ является локальньм и “плохим". Но при малых $h$ знаковая структура $D \pi^{*}$ в силу допущения утверждения 1 имеет вид $\Sigma$, т.е. является “хорошей". Противоречие, что и требовалось доказать.

Данное рассуждение позволяет обосновать и более общий результат о "наследовании". А именно, пусть семейство матриц, обладаюших свойством $\mu$, является полугруппой по умножению. Тогда если все $D L$ обладают свойством $\mu$, то и $D P$ обладает свойством $\mu$. Для иллюстрации приведем три примера.

ПРИмЕР 1. Для любой системы (2.1) $D L$ близок к тождественной матрице, поэтому определитель $D L$ положителен. Поскольку положительные числа образуют полугруппу, то и определитель $D P$ больше нуля. Значит, $P$ является диффеоморфизмом, сохраняющим ориентацию. 
ПРимеР 2. Пусть для некоторой трехмерной системы (2.1) знаковая структура $D L$ имеет вид $S$ (см. ниже). Хотя семейство таких матриц не замкнуто относительно операции умножения, оно порождает мультипликативную полугрупу типа $Z$ :

$$
S=\left(\begin{array}{ccc}
+1 & -1 & 0 \\
0 & +1 & +1 \\
0 & 0 & +1
\end{array}\right), \quad Z=\left(\begin{array}{ccc}
+1 & -1 & -1 \\
0 & +1 & +1 \\
0 & 0 & +1
\end{array}\right)
$$

Обьединение семейств $S$ и $Z$ также образует полугрупу $(S Z)$ по умножению. В силу принципа переноса $D P$ принадлежит $S Z$. На самом деле, $D P$ лежит в более узкой полугруппе $Z$. Действительно, представим $P$ в виде композиции двух полуотображений $Q_{1}$ и $Q_{2}$, определенных на $[0, T / 2]$ и $[T / 2, T]$ соответственно. Тогда $D Q_{1}$ и $D Q_{2}$ оба находятся в $S Z$ и, значит, для каждого полуотображения возможны два варианта: $D Q_{i}$ лежит или в $S$ или в $Z$. Непосредственно убеждаемся, что во всех четырех вариантах $D Q_{1} \cdot D Q_{2}$ принадлежит $Z$.

ПРИмеР 3. При отсутствии конкурентов динамика $i$-й популяции в системе (1.3) описьвается одномерньм уравнением в $\mathbb{R}_{+}$:

$$
\dot{x}_{i}=x_{i}\left[-1+\frac{\beta_{i}(t)}{V\left(x_{i}\right)}\right] \text {. }
$$

Пусть отображение $P: x_{i}^{0} \rightarrow x_{i}^{T}$ индуцировано уравнением $(2.2)$. Очевидно, $P(0)=0$. Далее, дифференциал каждого локального отображения (2.2) является положительным числом. Такие числа образуют полугруппу по умножению. Согласно принципу наследования устанавливаем $D P=d P / d x>0$, т.е. $P$ возрастает. Имеет место

ТЕОрема 1. При $\lambda_{i}>1$ в (2.2) существует единственная полохсительная $и$ глобально устойчивая в $\mathbb{R}_{+}$неподвижная точка отображения $P$. Данная точка принадлежит отрезку $\left[v\left(\lambda_{i}\right) \exp (-T), v\left(\lambda_{i}\right) \exp (T)\right]$.

ДокАЗАТЕЛЬСТво. Здесь для краткости изложения будем игнорировать индекс $i$. Переменная $x$ в $(2.2)$ изменяется не быстрее некоторых экспонент, поэтому для всех $t$ из $[0, T]$ справедливо

a) если $x^{0}$ мало, то $x^{t}$ мало и $V\left(x^{t}\right) \approx 1$; следовательно, при малых $x^{0}$ имеем $x^{T} \approx x^{0} \exp (\lambda T-T)$

б) если $x_{i}^{0}$ велико, то $x_{i}^{t}$ велико и $V\left(x^{t}\right) \approx \infty$; поэтому при больших $x^{0}$ имеем $x^{T} \approx x^{0} \exp (-T)$.

Следовательно, при $\lambda>1$ существует хотя бы одна положительная неподвижная точка $(r)$ у отображения $P$.

Оценим $r$. Пусть $x^{t}$ - положительное периодическое решение $(2.2)$, а $m$ и $M$ - его минимальное и максимальное значения. Очевидно, $m \leqslant r \leqslant M$. Так как $x^{t} \geqslant m$, имеем

$$
\frac{d x}{d t} \leqslant x\left[-1+\frac{\beta(t)}{V(m)}\right] .
$$

Проинтегрируем это неравенство по [0,T]. С учетом $x^{0}=x^{T}$ получаем $V(m) \leqslant \lambda$, т.е. $m \leqslant v(\lambda)$. Аналогично устанавливаем оценку $M \geqslant v(\lambda)$.

Так как $\beta>0$ и $V>0$, решение (2.2) убьвает не быстрее экспоненты, т.е. вьполняется неравенство $d x / d t>-x$. Справедлива 
ЛЕмма. Если $x^{0}=x^{T}$, mo $M / m<\exp (T)$.

ОБосновАНИЕ ЛЕмМЫ. В силу периодичности $x(t)$ можно считать, что максимум $x(t)$ реализуется при $t=0$, а минимум - при $t=a$, где $0<a<T$. Из $x(a)>x(0) \exp (-a)$ следует $m>M \cdot \exp (-T)$, что и требовалось доказать.

Теперь из неравенств $m \leqslant v(\lambda), M \geqslant v(\lambda)$ и $M / m<\exp (T)$ вытекают требуемые оценки $m \geqslant v(\lambda) \exp (-T)$ и $M \leqslant v(\lambda) \exp (T)$.

Последнее. Установим единственность и глобальную устойчивость $r$. Произведем в (2.2) замену $y=\ln x$. В новом уравнении

$$
\frac{d y}{d t}=-1+\frac{\beta(t)}{V[\exp (y)]}
$$

через $Q$ обозначим отображение Пуанкаре: $y^{0} \rightarrow y^{T}$. Очевидно, каждой положительной неподвижной точке $P$ соответствуют “своя" неподвижная точка $Q$ в $R$. Заметим, что все дифференциалы локального отображения $(2.3)$ оказьваются числами из $(0,1)$. Данные числа образуют полугруппу по умножению. В частности, получаем $d Q / d y<1$ для всех $y$. Поэтому $Q$ - сжимающее отображение и, значит, оно может иметь не более одной неподвижной точки. Если такая точка существует, то она, разумеется, глобально устойчива, что и требовалось доказать.

При $\lambda \leqslant 1$ (т.е. в среднем рождаемость не больше смертности) отображение $P$ не имеет положительных неподвижных точек, и, значит, такая популящия вымирает. Поэтому будем считать, что в системе (1.3) для всех $i$ вьполняется условие $\lambda_{i}>1$.

3. Динамика двух конкурентов с матрицей $\Sigma(1,-1)$. Содержание данного пункта можно трактовать как четвертьй пример использования знак-инвариантных матриц. Рассмотрим сначала систему двух конкурентов в общем виде

$$
\dot{x}_{1}=x_{1} g_{1}\left(x_{1}, x_{2}, t\right), \quad \dot{x}_{2}=x_{2} g_{2}\left(x_{1}, x_{2}, t\right)
$$

и попытаемся из нее извлечь максимум "полезной информации". Затем для установления некоторых тонких, но необходимых свойств конкретизируем (3.1) в виде (1.3).

Напомним поведение правых частей системы (3.1): $\partial g_{i} / \partial x_{j}<0$ для всех $i, j$ и каждая $g_{i}$ является $T$-периодической функцией по последнему аргументу. Легко видеть, что дифференциал локального отображения (3.1) представляется знак-инвариантной структурой $\Sigma(1,-1)$ и, значит, знаковая структура дифференциала глобального отображения Пуанкаре

$$
x_{1}^{T}=P_{1}\left(x_{1}^{0}, x_{2}^{0}\right), \quad x_{2}^{T}=P_{2}\left(x_{1}^{0}, x_{2}^{0}\right)
$$

имеет вид $\Sigma(1,-1)$. Каждая функция $P_{i}$ монотонно возрастает по “своей" переменной $\left(x_{i}\right)$ и убывает по “чужой” переменной.

На точках плоскости $\mathbb{R}_{+}^{2}$ зададим следующее отношение частичного порядка [8]. Пусть даны две точки $A=\left(a_{1}, a_{2}\right)$ и $B=\left(b_{1}, b_{2}\right)$; тогда положим $A \ll B$, если выполняются неравенства $a_{1} \leqslant b_{1}$ и $a_{2} \geqslant b_{2}$. Очевидно, соотношение $A \ll B$ эквивалентно принадлежности вектора $(B-A)$ четвертому квадранту $(K)$ плоскости $\mathbb{R}^{2}$. Из приведенных свойств монотонности $P$ легко получаем следующее утверждение. 
Лemma 1. Eсли $A \ll B$, mo и $P(A) \ll P(B)$.

Пусть заданы две точки $A$ и $B$, связанные отношениями $A \ll B$. Тогда рассмотрим "конусный отрезок" П $(A, B)$ - множество "промежуточных" точек $\mathrm{X}$, удовлетворяющих одновременно условиям $A \ll X$ и $X \ll B$. Геометрически $\Pi(A, B)$ представляет собой прямоугольник, в котором "слабал" точка $A$ находится на северо-востоке, а "сильная" точка $B$ лежит на юго-западе.

Лемма 2. Под действием $P$ образ конусного отрезка $\Pi(A, B)$ вложен в конусный отрезок $\Pi(P(A), P(B))$.

Последнее свойство позволяет доказывать устойчивость равновесия $(r)$ по следующей известной схеме. Пусть вокруг $r$ удается построить семейство конусных отрезков $\left\{\Pi_{i}\right\}$, которые с ростом $i$ стягиваются в точку $r$. Если для всех $i$ образ $P\left(\Pi_{i}\right)$ вложен в $\Pi_{i+1}$, то, разумеется, $r$ устойчиво.

Теперь наступил момент, ког да следует конкретизировать систему (3.1) по схеме (1.3). А именно, положим

$$
\dot{x}_{1}=x_{1}\left[-1+\frac{\beta_{1}(t)}{V(S)}\right], \quad \dot{x}_{2}=x_{2}\left[-1+\frac{\beta_{2}(t)}{V(S)}\right],
$$

где $S=x_{1}+x_{2}$. Напомним, что $\lambda_{i}$ (среднее за период значение коэффициента $\beta_{i}$ ) больше 1 для всех $i$. В работе [9] на примере схемы Контуа была установлена

Теорема 2. Пусть выполняется условие запаса

$$
v\left(\lambda_{1}\right)>v\left(\lambda_{2}\right) \exp (T) .
$$

Тогда в системе (3.2) первый конкурент вытесняет второго.

Суть обоснования данной теоремы заключается в следующем.

1. На основе системы (3.2) определим в $\mathbb{R}_{+}^{2}$ два множества полуравновесий $E_{1}$ и $E_{2}$, где $E_{i}=\left\{\left(x_{1}^{0}, x_{2}^{0}\right) \mid x_{i}^{0}=x_{i}^{T}>0\right\}$. Каждая кривая $E_{i}$ "притягивает" к себе векторное поле $P$ по своей координате. Самое главное, что при выполнении условия запаса кривая $E_{1}$ располагается вьше $E_{2}$ в $\mathbb{R}_{+}^{2}$ (см. рис. 1$)$.

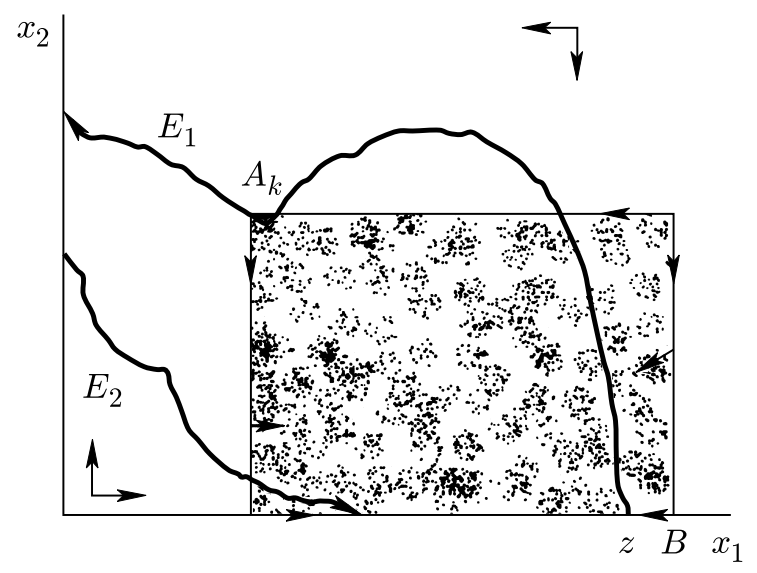

Р ис. 1. К обоснованию признака отбора в системе (3.2). Пояснения в тексте 
2. Пусть $r_{1}$ - точка пересечения $E_{1}$ с осью абсцисс. С помощью “высокой” кривой $E_{1}$ построим "матрешку" - $k$-параметрическое семейство стягивающихся конусных отрезков $\{\Pi(k)\}$. Здесь подвижная “слабая" вершина $A_{k}$ лежит на кривой $E_{1}$ и имеет координаты $\left\langle k r_{1}, E_{1}\left(k r_{1}\right)\right\rangle$. Напротив, “сильная" вершина $B$ закреплена в точке $\left\langle r_{1}+\zeta, 0\right\rangle$, где константа $\zeta>0$. При $k=0$ имеем наибольший прямоугольник П $(0)$, а при $k=1-$ наименьший прямоугольник $\Pi(1)$, которьй вырождается в отрезок $\left[r_{1}, r_{1}+\zeta\right]$, лежащий на оси абсцисс.

3. Под действием $P$ ○ $P$ каждый “большой” конусный отрезок П $(k)$ переходит в “малый” $\Pi(m)$, где $m>k$. Отсюда стандартным образом заключаем, что точка $\left\langle r_{1}, 0\right\rangle$ глобально устойчива в $\mathbb{R}_{+}^{2}$. Это означает, что второй конкурент вымирает.

4. Динамика многих конкурентов с матрицей $\Sigma(1, \ldots, 1)$. Покажем, что и в случае $n \geqslant 3$ конкурентов константа запаса также равна $\exp (T)$. Напомним исходную систему:

$$
\dot{x}_{i}=x_{i}\left[-1+\frac{\beta_{i}(t)}{V\left(x_{1}+\cdots+x_{n}\right)}\right],
$$

где $i=1, \ldots, n$. Здесь наглядная геометрическая идея рассуждений остается, по сути, прежней. Сначала установим, что при некоторых ограничениях на параметры $\left\{\lambda_{i}\right\}$ поверхность $E_{1}$ лежит выше всех остальных поверхостей $E_{i}$, где

$$
E_{i}=\left\{\left(x_{1}^{0}, \ldots, x_{n}^{0}\right) \mid x_{i}^{0}=x_{i}^{T}>0\right\} .
$$

Затем с помощью $E_{1}$ построим инвариантное (под действием $P$ ) семейство областей, стягивающихся к точке $\left(r_{1}, 0, \ldots, 0\right)$. Однако техническая реализация этого подхода сталкивается с принципиальными трудностями, так как при $n \geqslant 3$ знаковая структура дифференциала локального отображения (4.1) не является знак-инвариантной. Поэтому сдвиг-отображение $P$ может оказаться “плохим", поскольку для него нельзя гарантировать "хорошие" монотонные свойства.

Тем не менее здесь возможен следующий окольный путь. В (4.1) заменим время на противоположное $(t \rightarrow-t)$. Эта система действует в том же фазовом пространстве $\mathbb{R}_{+}^{n}$, но, чтобы не возникало путаницы, в новой системе переименуем переменные

$$
\dot{y}_{i}=y_{i}\left[1-\frac{\beta_{i}(-t)}{V\left(y_{1}+\cdots+y_{n}\right)}\right],
$$

где $i=1, \ldots, n$. С биологической точки зрения данная система описывает динамику симбиоза, в котором популяции оказывают положительное влияние друг на друга.

Сдвиг-отображение $(Q)$ системы (4.2) оказывается "хорошим". Действительно, знаковая структура каждого дифференциала локального отображения (4.2) является знакинвариантной матрицей $\Sigma(1, \ldots, 1)$. Значит, для любого $i$ в координатном представлении $y_{i}^{T}=Q_{i}\left(y_{1}^{0}, \ldots, y_{n}^{0}\right)$ функция $Q_{i}$ возрастает по каждой переменной.

Очевидно, $Q$ - обратное отображение к $P$. Поэтому существует тесная взаимосвязь между системами (4.2) и (4.1). Следовательно, анализ одной из них доставляет полезную информацию и о другой.

Для каждого $i$ в (4.2) определим множество полуравновесий

$$
J_{i}=\left\{\left(y_{1}^{0}, \ldots, y_{n}^{0}\right) \mid y_{i}^{0}=y_{i}^{T}>0\right\} .
$$

Разумеется, $J_{i}$ можно также задать соотношением $y_{i}=Q_{i}\left(y_{1}, \ldots, y_{n}\right)$. 
Свойство 1. При и только при $\lambda_{i}>1$ множество $J_{i}$ непусто.

ДокАЗАТЕЛЬСтво. Для определенности положим $i=1$. Так как $V>1$ при $y_{1}+\cdots$ $+y_{n}>0$, имеет место неравенство

$$
\frac{d y_{1}}{d t}>y_{1}\left[1-\beta_{1}(-t)\right]
$$

Следовательно, $y_{1}^{T}>y_{1}^{0} \exp \left[T-T \lambda_{1}\right]$. Значит, при $\lambda_{1} \leqslant 1$ всегда получаем $y_{1}^{T}>y_{1}^{0}$. Поэтому при $\lambda_{1} \leqslant 1$ не существует $J_{1}$.

Пусть теперь $\lambda_{1}>1$. Возьмем две точки $A=\left(m, y_{2}^{0}, \ldots, y_{n}^{0}\right)$ и $B=\left(M, y_{2}^{0}, \ldots, y_{n}^{0}\right)$, в которых все $y_{2}^{0}, \ldots, y_{n}^{0}$ - малые положительные числа. Поскольку в системе (4.2) переменные растут и убывают не слишком быстро, имеем, в частности, что $y_{i}^{t}$ малы при всех $t$ из $[0, T]$ для $i>1$. Поэтому динамика переменной $y_{1}$ примерно описывается уравнением $d y_{1} / d t \approx y_{1}\left[1-\beta_{1}(-t) / V\left(y_{1}\right)\right]$. Рассмотрим два случая.

1) Если $y_{1}^{0}=M$ и $M$ велико, то $y_{1}^{t}$ велико для всех $t$ из $[0, T]$. Значит, $d y_{1} / d t \approx y_{1}$, поэтому в точке $B$ имеем $y_{1}^{T}>y_{1}^{0}$.

2) Если $y_{1}^{0}=m$ и $m$ мало, то $y_{1}^{t}$ мало для всех $t$ из $[0, T]$. Значит, $d y_{1} / d t \approx y_{1}\left[1-\beta_{1}(-t)\right]$ и, следовательно, $y_{1}^{T} \approx y_{1}^{0} \exp \left(T-T \lambda_{1}\right)$. Поэтому при $\lambda_{1}>1$ в точке $A$ получаем $y_{1}^{T}<y_{1}^{0}$.

Наконец, из соображений непрерывности заключаем, что в некоторой промежуточной точке $\left(y_{1}^{0}\right)$ отрезка $[m, M]$ имеет место $y_{1}^{T}=y_{1}^{0}$. Иными словами, множество $J_{1}$ непусто, что и требовалось доказать.

Отметим следуюшие полезные "двойственные" свойства.

1) $J_{i}=P\left(E_{i}\right)$ и $Q\left(J_{i}\right)=E_{i}$. Поэтому если $J_{1}$ и $J_{i}$ не пересекаются, то $E_{1}$ и $E_{i}$ не пересекаются.

$2)$ Пусть $Q$ переводит точку $A$ в точку $B$. Тогда $P$ переводит $B$ в $A$. В частности, векторное поле в системе (4.1) получается путем обращения стрелок в векторном поле (4.2).

3) Неподвижная точка $P$ является неподвижной точкой и $Q$.

Назовем некоторую поверхность $F\left(y_{1}, \ldots, y_{n}\right)=0$ монотонной в $\mathbb{R}_{+}^{n}$, если в каждой ее точке все частные производные $F$ положительны. Тогда можно выразить, например, $y_{1}$ через остальные переменные $\varphi\left(y_{2}, \ldots, y_{n}\right)$, при этом все частные производные $\varphi$ отрицательны.

Свойство 2. Поверхность $J_{i}$ является монотонной.

ДоКАЗАТЕЛЬСТво. Построим вспомогательную систему, производя в (4.2) монотонную замену $y_{i}=\exp \left(z_{i}\right)$ для всех $i$ :

$$
\dot{z}_{i}=1-\frac{\beta_{i}(-t)}{V\left[\exp \left(z_{1}\right)+\cdots+\exp \left(z_{n}\right)\right]} .
$$

Продемонстрируем сначала монотонность, для примера, $J_{1}$ в координатах $\left\{z_{i}\right\}$. Для этого рассмотрим дифференциал локального отображения (4.3). Его знаковая структура состоит из одних плюсов, причем на диагонали "сидят" элементы, большие единицы. 
Такие матрицы образуют полугруппу по умножению, и, значит, дифференциал глобального отображения $\widetilde{Q}=\left(\widetilde{Q}_{1}, \ldots, \widetilde{Q}_{n}\right)$ (отображение Пуанкаре в $\left.(4.3)\right)$ обладает тем же свойством. В частности, $d \widetilde{Q}_{1} / d z_{1}>1$. Далее, множество $J_{1}$ задается уравнением

$$
F\left(z_{1}, \ldots, z_{n}\right)=\widetilde{Q}_{1}\left(z_{1}, \ldots, z_{n}\right)-z_{1}=0 .
$$

В силу сказанного все частные производные $F$ положительны. Поэтому в переменных $\left\{z_{i}\right\}$, а значит, и в переменных $\left\{y_{i}\right\}$ поверхность $J_{1}$ монотонна, что и требовалось доказать.
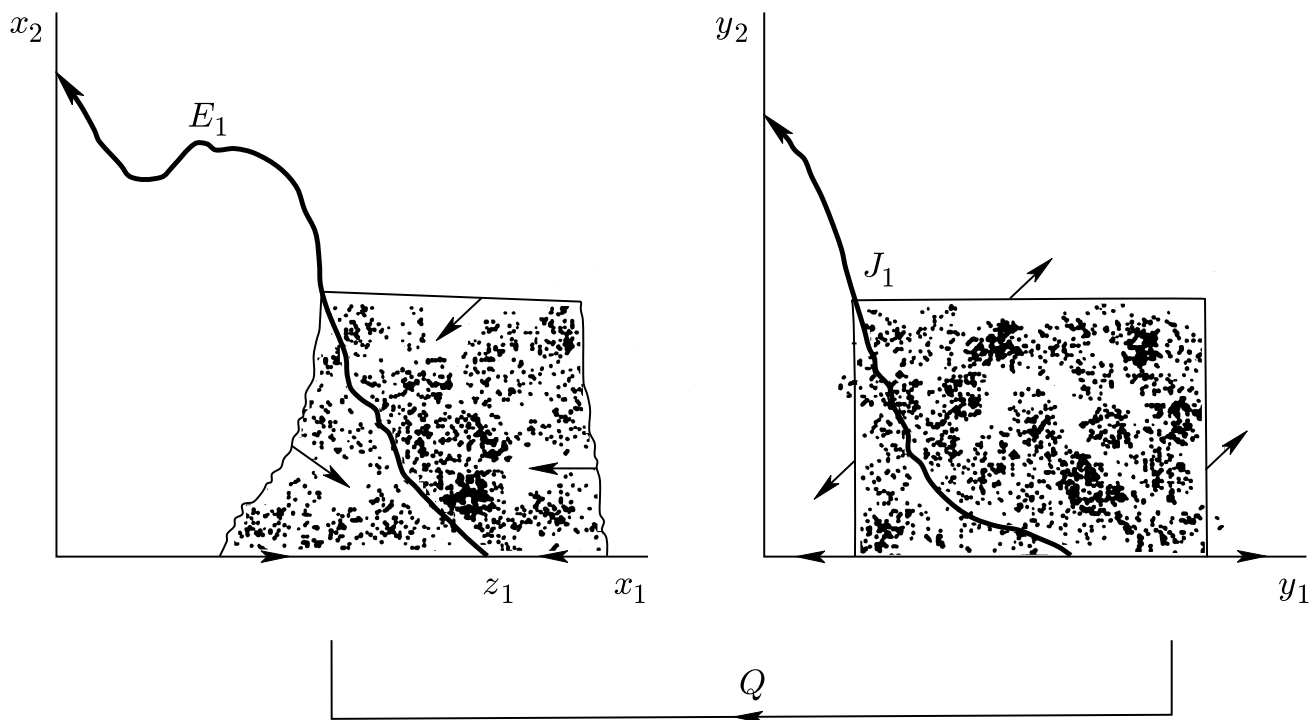

Рис. 2. Двумерная иллюстрация взаимосвязи фазовых портретов систем (4.1) и (4.2). Пояснения в тексте

Каждая монотонная поверхность $J_{i}$ разбивает $\mathbb{R}_{+}^{n}$ на две “свои" области - "верхнюю" (неограниченную) и "нижнюю” (ограниченную) (см. рис. 2). Приведем аналитическую характеристику этих областей. А именно, точка $\left(y_{1}, \ldots, y_{n}\right)$ принадлежит:

а) "нижней" области при $y_{i} \geqslant Q_{i}\left(y_{1}, \ldots, y_{n}\right)$; тогда $y_{i}^{T} \leqslant y_{i}^{0}$;

б) "верхней" области при $y_{i} \leqslant Q_{i}\left(y_{1}, \ldots, y_{n}\right)$; тогда $y_{i}^{T} \geqslant y_{i}^{0}$.

Грубо говоря, $J_{i}$ “отталкивает" от себя векторное поле (4.2). Значит, $E_{i}$ "притягивает" к себе векторное поле системы (4.1).

Свойство 3. Если выполняется условие запаса

$$
v\left(\lambda_{1}\right)>v\left(\lambda_{i}\right) \exp (T) \quad \text { для всех } \quad i>1,
$$

то $J_{1}$ расположсена выше всех остальных поверхностей $J_{i}$ в $\mathbb{R}_{+}^{n}$.

ДоказАТЕЛьСтво. Так как $\beta_{i}>0$ и $V>0$, в (4.2) имеем $d y_{i} / d t<y_{i}$ для всех $i$. Для таких функций выполняется следующая относительная оценка минимума $\left(m_{i}\right)$ и максимума $\left(M_{i}\right)$ на $[0, T]$. 
ЛЕММА О КРАЯХ. Если $y_{i}^{0} \leqslant y_{i}^{T}$, mo $M_{i} / m_{i} \leqslant \exp (T)$.

ОБосновАнИЕ ЛЕмМЫ. Пусть значение $m_{i}$ достигается в точке $t=a$, а значение $M_{i}-$ в точке $t=b$. Возможны следующие варианты расположения точек $a$ и $b$ на отрезке $[0, T]$.

1) $a<b$. Поскольку $y_{i}$ растет не быстрее экспоненты, имеем $m_{i} \exp (b-a) \geqslant M_{i}$. Значит, и $M_{i} / m_{i} \leqslant \exp (T)$.

2) $b<a$. Аналогично, выполняются неравенства $y_{i}^{0} \exp (b) \geqslant M_{i}$ и $m_{i} \exp (T-a) \geqslant y_{i}^{T}$. Значит, $M_{i} / m_{i} \leqslant\left(y_{i}^{0} / y_{i}^{T}\right) \exp (T+b-a)$. В силу сделанных допущений окончательно имеем $M_{i} / m_{i} \leqslant \exp (T)$, что и требовалось.

Предположим противное: некоторые ("плохие") поверхности $J_{i}$ пересекают или лежат вьше $J_{1}$ в $\mathbb{R}_{+}^{n}$. Определим в $\mathbb{R}_{+}^{n}$ область- "клин" $(W)$ : каждая точка $W$ одновременно лежит выше всех $J_{2}, \ldots, J_{n}$. Клин всегда непуст. Легко сообразить, что при сделанном допущении клин пересекает или полностью находится вьше $J_{1}$ в $\mathbb{R}_{+}^{n}$. Установим противоречивость каждого из этих случаев.

1. Клин пересекает $J_{1}$. Тогда в $\mathbb{R}_{+}^{n}$ существует $Y^{*}=\left(y_{1}^{0}, \ldots, y_{n}^{0}\right)$ - точка пересечения $J_{1}$ c, например, $J_{2}$, которая лежит вьше всех остальных поверхностей $J_{3}, \ldots, J_{n}$.

Рассмотрим решение (4.2), выходящее из данной точки. Тогда имеем $y_{1}^{0}=y_{1}^{T}, y_{2}^{0}=y_{2}^{T}$ и $y_{i}^{0} \leqslant y_{i}^{T}$ для всех $i>2$. Обозначим $m=m_{1}+\cdots+m_{n}$ и $M=M_{1}+\cdots+M_{n}$. Из леммы о краях легко выводим $M / m \leqslant \exp (T)$. Сопоставим следующие результаты.

а) Для положительных периодических переменных $y_{1}$ и $y_{2}$ покажем, что точки $v\left(\lambda_{1}\right)$ и $v\left(\lambda_{2}\right)$ одновременно принадлежит отрезку $[m, M]$. Действительно, из $V\left(y_{1}+\cdots+y_{n}\right)>$ $V(m)$ для $i=1,2$ получаем

$$
\frac{d y_{i}}{d t}>y_{i}\left[1-\frac{\beta_{i}(-t)}{V(m)}\right]
$$

После интегрирования этого неравенства в промежутке $[0, T]$ находим

$$
y_{i}^{T}>y_{i}^{0} \exp \left[T-T \frac{\lambda_{i}}{V(m)}\right] \text {. }
$$

Из $y_{i}^{0}=y_{i}^{T}$ следует $m<v\left(\lambda_{i}\right)$.

Аналогично, из $V\left(y_{1}+\cdots+y_{n}\right)<V(M)$ следует $M>v\left(\lambda_{i}\right)$ для $i=1,2$. Значит, $\rho=v\left(\lambda_{1}\right) / v\left(\lambda_{2}\right) \leqslant M / m$. Отсюда следует $\rho \leqslant \exp (T)$;

б) Из условия запаса получаем $\rho=v\left(\lambda_{1}\right) / v\left(\lambda_{2}\right)>\exp (T)$. Последние два неравенства противоречат друг другу.

2. Клин находится выше $J_{1}$. K этому случаю также отнесем ситуации, когда имеет место касание клина и $J_{1}$ на границе $\mathbb{R}_{+}^{n}$. Рассмотрим $k$-параметрическое семейство систем

$$
\dot{y}_{1}=y_{1}\left[1-\frac{\beta_{1}(-t)}{V(S)}\right], \quad \dot{y}_{2}=y_{2}\left[1-k \frac{\beta_{2}(-t)}{V(S)}\right], \quad \ldots, \quad \dot{y}_{n}=y_{n}\left[1-k \frac{\beta_{n}(-t)}{V(S)}\right],
$$

где $S=y_{1}+\cdots+y_{n}$ и параметр $k$ принадлежит $(0,1]$. Очевидно, при $k=1$ система $(4.4)$ совпадает с (4.2). Так как $v$ монотонно возрастает, при любом $k<1$ в системе (4.4) выполняется условие запаса

$$
v\left(\lambda_{1}\right)>v\left(k \lambda_{i}\right) \exp (T) \quad \text { для всех } i>1 .
$$


Начнем последовательно уменьшать $k$ от 1 до 0; тогда происходит непрерьвная деформация поверхностей $J_{1}(k)$ и всех остальных $J_{i}(k)$. Пусть $W(k)$ - клин, построенньй из поверхностей $J_{2}(k), \ldots, J_{n}(k)$. Разумеется, область $W(k)$ меняется непрерьвным образом как функция $k$. Теперь заметим следующее:

a) $W(1)$ находится выше $J_{1}$;

б) при малых $k(k \rightarrow+0)$ поверхность $J_{1}(k)$ не исчезает, а остальные $J_{i}(k)$ исчезают; значит, $W(+0)$ заполняет все $\mathbb{R}_{+}^{n}$ и, в частности, включает в себя $J_{1}$.

Проведем в $\mathbb{R}_{+}^{n}$ “биссектрису" (прямую $y_{1}=y_{2}=\cdots=y_{n}$ ). Точку пересечения $W$ и биссектрисы назовем “центром” границы клина.

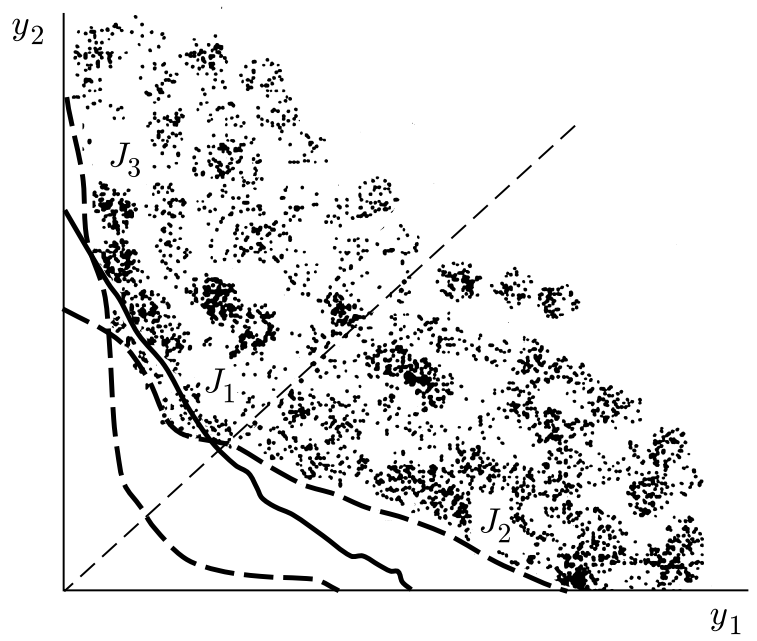

Рис. 3. К доказательству свойства 3. Момент встречи центра клина и поверхности $J_{1}$ (двумерная иллюстрация)

В силу а) и б) при некотором промежуточном $k^{*}$ клин касается своим центром поверхности $J_{1}$ в какой-то точке $Y^{*}$ из $\mathbb{R}_{+}^{n}$ (см. рис. 3 ). По сути, в $Y^{*}$ поверхность $J_{1}$ пересекается с некоторой $J_{i}$ и $Y^{*}$ находится выше остальных поверхностей. Такая ситуация полностью соответствует случаю 1 , которьй нереализуем, что и требовалось доказать.

Пусть монотонная поверхность $J_{1}$ задается уравнением $y_{1}=\varphi\left(y_{2}, \ldots, y_{n}\right)$ и пересекает ось $y_{1}$ в точке $\left(r_{1}, 0 \ldots, 0\right)$. Разумеется, $r_{1}=\varphi(0, \ldots, 0)$. В замькании $\mathbb{R}_{+}^{n}$ построим $k$-параметрическое семейство вложенных призм

$$
\pi(k)=\left\{\left(y_{1}, \ldots, y_{n}\right) \mid k r_{1} \leqslant y_{1} \leqslant r_{1}+\zeta \text { и } \varphi\left(y_{2}, \ldots, y_{n}\right) \geqslant k r_{1}\right\},
$$

где $k$ изменяется от 0 до 1 ; константа $\zeta>0$ (см. рис. 2). Очевидно, самая маленькая призма $\pi(1)$ вырождается в отрезок $\left[r_{1}, r_{1}+\zeta\right]$.

Назовем боковой поверхностью призмы те ее точки, к которьм можно подойти снаружи из $\mathbb{R}_{+}^{n}$. Возможны следующие три варианта положения точки $\left(y_{1}^{0}, \ldots, y_{n}^{0}\right)$ на боковой поверхности $\pi(k)$.

1) Правая поперечная граница, т.е. $y_{1}^{0} \geqslant r_{1}+\xi$. Тогда данная точка находится вьше $J_{1}$ и, значит, $y_{1}^{T}>y_{1}^{0}$. Поэтому точка $Y^{T}=\left(y_{1}^{T}, \ldots, y_{n}^{T}\right)$ уходит вправо и оказьвается вне $\pi(k)$. 
2) Продольная граница, т.е. $\varphi\left(y_{2}^{0}, \ldots, y_{n}^{0}\right)=k r_{1}$ и $y_{1}^{0} \geqslant 0$. При условии запаса данная точка находится вьше $J_{i}$. Если $y_{i}^{0}=0$, то и $y_{i}^{T}=0$. А если $y_{i}^{0}>0$, то $y_{i}^{T}>y_{i}^{0}$. Здесь, разумеется, не все $y_{i}^{0}=0$ для $i>1$. Поскольку $\varphi-$ убьвающая функция, имеем $\varphi\left(y_{2}^{T}, \ldots, y_{n}^{T}\right)<k r_{1}$. Следовательно, точка $Y^{T}$ уходит вверх и оказьвается вне $\pi(k)$.

3) Левая поперечная гранища, т.е. $y_{1}^{0}=k r_{1}$. Тогда данная точка находится ниже $J_{1}$ и поэтому $y_{1}^{T}<y_{1}^{0}$. Значит, точка $Y^{T}$ уходит влево и оказывается вне $\pi(k)$.

Таким образом, доказано

Свойство 4. Пусть поверхность $J_{1}$ расположена выше всех остальных $J_{i}$. Тогда в точках боковой поверхности призмы $\pi(k)$ векторное поле отобрахения $Q$ направлено наружу.

Иными словами, если $Y$ принадлежит границе $\pi(k)$, то $Q(Y)$ принадлежит границе $\pi(m)$, где $k>m$. По существу, вся подготовительная работа по построению фазового портрета системы (4.1) проделана с помощью “хорошего" отображения $Q$. Теперь нетрудно заключительный шаг провести в рамках исходного “плохого" отображения $P$.

ТЕОРема 3. При выполнении условия запаса в системе (4.1) первый конкурент вытесняет остальных.

ДокАзАТЕЛЬСТво. Обозначим через $\Pi(k)$ образ $\pi(k)$ под действием $Q$. При условии запаса самая большая область $\Pi(+0)$ включает в себя все $E_{i}$, поскольку каждое $J_{i}$ переходит в $E_{i}$. С увеличением $k$ область $\Pi(k)$ сокращается и, наконец, П(1) вырождается в отрезок $\left[r_{1}, r_{1}+\xi\right]$, где константа $\xi>0$.

Векторное поле $P$ на гранище П $(k)$ направлено строго вовнутрь. В противном случае пусть $X$ и $P(X)$ лежат соответственно на поверхностях $\Pi(k)$ и $\Pi(m)$, где $k \geqslant m$. Тогда $Q$ преобразует $P(X)$ в $X$ и согласно свойству 4 имеем $k<m$. Противоречие.

Пусть $X^{0}$ - произвольная точка $\mathbb{R}_{+}^{n}$. Она порождает последовательность $X^{s+1}=$ $P\left(X^{s}\right)$. Нетрудно показать, что какая-то точка $X^{s}$ оказывается в некотором $\Pi(k)$ (удобно считать, что это $X^{0}$ ). На орбите данной последовательности определим следующий непрерьвньй неотрицательньй функционал $(\Lambda)$ ляпуновского типа: $е c л u X^{s}$ лежит на границе $\Pi\left(k_{s}\right)$, то $\Lambda\left(X^{s}\right)=1-k_{s}$. Положим $\rho_{s}=\Lambda\left(X^{s}\right)$. Самое главное, что векторное поле $P$ направлено вовнутрь $\Pi\left(k_{s}\right)$, поэтому $\rho_{s+1}<\rho_{s}$.

Пусть $\rho^{*}$ - предел убьвающей последовательности положительных чисел $\left\{\rho_{s}\right\}$. Покажем, что $\rho^{*}=0$. Предположим противное: $\rho^{*}>0$. Тогда на боковой поверхности призмы $\Pi^{*}=\Pi\left(1-\rho^{*}\right)$ находится $X^{*}$ - одна из предельных точек последовательности $\left\{X^{s}\right\}$. Рассмотрим действие $P$ на $X^{*}$. С одной стороны, векторное поле $P$ "загоняет" $P\left(X^{*}\right)$ вовнутрь $\Pi^{*}$, а с другой стороны, - все $\left\{X^{s}\right\}$ находятся снаружи (в крайнем случае, на границе) $\Pi^{*}$. Здесь в силу непрерьвности $P$ данная последовательность “удерживает" $P\left(X^{*}\right)$ снаружи $\Pi^{*}$. Противоречие.

Значит, $\rho^{*}=0$ и, следовательно, предельная точка $\left\{X^{s}\right\}$ оказьвается в $\Pi(1)=$ $\left[r_{1}, r_{1}+\xi\right]$. Поскольку $\xi$ - любое положительное число, точка $R=\left(r_{1}, 0, \ldots, 0\right)$ глобально устойчива в $\mathbb{R}_{+}^{n}$, что и требовалось доказать.

ЗАключениЕ. Универсальные константы запаса обнаружены и в других классах моделей конкуренции (например, вольтерровских [10]). Вероятно, в системах "все против всех" конкурентное давление на первого участника всех остальных ослабляется из-за их междуусобной конкуренции. 


\section{СПИСОК ЦИТИРОВАННОЙ ЛИТЕРАТУРЫ}

[1] Розоноэр Л.И., Седых Е. И. О механизмах эволюции самовоспроизводящихся систем. I // Автоматика и телемеханика. 1979. № 2. С. 110-119.

[2] Ильичев В. Г. Фрагмент математической теории конкуренции биологических видов в переменной среде // Дифференц. уравнения. 1991. Т. 27. № 3. С. 437-447.

[3] Ильичев В.Г. Дельта-функции и теория биологической конкуренции в переменной среде // Автоматика и телемеханика. 1996. № 11. С. 115-127.

[4] Vance R. R., Coddington E. A. A nonautonomus model of population growth // J. Math. Biology. 1989. V. 27. № 5. P. 491-506.

[5] Ильичев В. Г. О технологии построения имитационных моделей экологических систем. Механизмы адаптации // Матем. моделирование. 1992. Т. 4. № 3. С. 11-19.

[6] Contois D. E. Kinetics of bacterial growth, relationship between population density and specific growth rate of continuous culture // Gen. Microbiol. 1959. №1-2. P. 40.

[7] Ильичев В.Г., Ильичева О.А. Дискретные модели и знак-инвариантные структуры матриц // Изв. РАН. Теория и системы управления. 1998. № 4. С. 110-117.

[8] Красносельский М. А., Забрейко П. П. Геометрические методы нелинейного анализа. М.: Наука, 1975.

[9] Ильичев В. Г. Неожиданные свойства конкуренции биологических видов в переменной среде // Автоматика и телемеханика. 1990. №9. С. 34-44.

[10] Ильичев В.Г. Дельта-функции и исследование экологических моделей Вольтерра в переменной среде // Изв. вузов. Матем. 1998. № 4. С. 23-33.

НИИ механики и прикладной математики

Поступило

при Ростовском госуниверситете

12.12.1997

Исправленный вариант

01.03.1999 